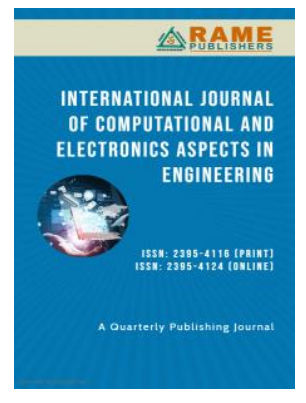

Samyak S. Nagrare ${ }^{1}$

2021samyak@gmail.com

Payal N. Kanchanwar ${ }^{2}$ Kanchanwarpayal1@gmail.com

Prashant V. Patle ${ }^{3}$ ppatle528@gmail.com

Varsha V. Ambule ${ }^{4}$ varsha.var53@gmail.com

Department of Information Technology, Smt. Radhikatai Pandav College of Engineering, Nagpur, India

\title{
Real-Time Smart Face Security Estimation
}

Abstract - The intricacies of Sighthound's absolutely computerized age, gender, and emotion identity set of rules are defined. Our machine is constructed on numerous deep convolutional neural networks, which aren't simplest computationally green however additionally produce modern effects on quite a few aggressive benchmarks. We accumulated significant labelled datasets from all around the international to construct our progressive deep networks. We describe a methodology and an algorithm to estimate the real-time age, emotion, and gender of a human by investigating face images on a webcam. Here we discuss the $\mathrm{CNN}$-based architecture to design a real-time model. Security system Emotion, age, and gender detection of facial images in webcams play an important role in many applications like security control forensics, data analysis, video observation, and human-computer interaction.

Keywords - Machine Learning, Deep Learning, Convolutional Network, CNN, Gender Estimator, Age Predictor, Emotion Detector, Python.

\section{INTRODUCTION}

One of the most active areas in facial innovation is facial features such as gender, age, and emotion recognition. Research has been done using deep learning methods such as ANN, CNN to determine age, gender estimation, and emotion identification. Human Facial expression depicts human feelings. Human tends to change feelings, perhaps due to their mental or psychological health conditions, in the course of the lifespan.

Although people are brimming with various feelings, modern psychology characterizes six basic facial happiness expressions, sadness, fear surprise, fear, disgust, and anger as widespread feelings. Facial muscles exercise to assist with perceiving an individual's feelings. Fundamental facial consideration features are mouth, eyebrows, nose, and eyes.

\section{Research Article}

First Online on - 11 June 2021

(C) 2021 RAME Publishers

This is an open access article under the CC BY 4.0 International License https://creativecommons.org/licenses/by/4.0/

Cite this article - Samyak S. Nagrare, Payal N. Kanchanwar, Prashant V. Patle, Varsha V. Ambule, "Real-Time Smart Face Security Estimation", International Journal of Computational and Electronic Aspects in Engineering, RAME Publishers, vol. 2, issue 2, pp. 18-23, 2021. https://doi.org/10.26706/ijceae.2.2.20210408
An architecture based on the Convolution Neural network $(\mathrm{CNN})$ proposed to use here for age, emotion classification, and gender classification. This is one of the well-known deep artificial neural networks. Convolutional Neural Network-based design models are broadly utilized in classification tasks because of their remarkable execution in facial investigation and emotion detection.

The Convolutional Neural Network includes new Feature extraction which extracts Features corresponding to age, gender, emotion. Furthermore, CNN includes Feature classification which classifies facial images into the correct age group gender and emotion such as happy, sad, angry, neutral [1,2].

\section{GENDER AND AgE Classification}

Gender and age classification is the facial features that play a significant role in social interaction. Age and gender classification in face images play important task for example, access control, low authorization, visual observation and so on [3].

Emotion detection on face images can communicate and impact individual's feelings. It is intriguing and to basic comprehend the feelings passed on by the images and how the visual content of the image implies emotion [4]. 


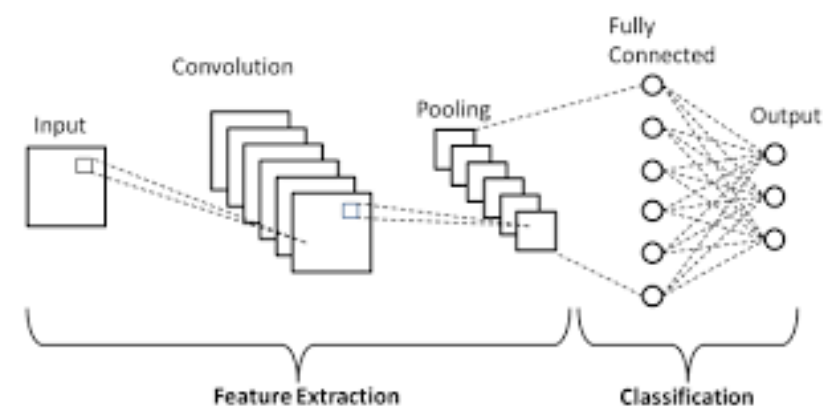

Figure 1: System Basic CNN architecture.

\section{PROPOSED Methodology}

The primary objective of the proposed methodology is to recognize the gender and age range with emotion from the human face images utilizing the set of facial features in real time application. Feature extraction from face images is an important part of this method. In here, Fig-1 represent to show the flow of step to this methodology [5].

\section{A. Pre-Possessing}

The pre-processing stage can improve the quality of the input image and find the data of interest by eliminating noise and smoothing the image. It eliminates image redundancy without image details. Pre-processing also includes filtering and normalizing the image to produce a uniform size and rotated image.

\section{B. Face Detection}

The face detection process is to extract the face area from the background of Input images with various lighting conditions and complex backgrounds can be confusing and fail to recognize these expressions. It involves segmentation and extraction of facial features from the uncontrolled background.

\section{Feature Extraction}

In object detection feature extraction plays important role. Here, it includes shapes, movement, color, the texture of the facial image. It extracts meaningful information of an image compared to the original image. Feature extraction greatly reduces information of image, which has advantages in storage.

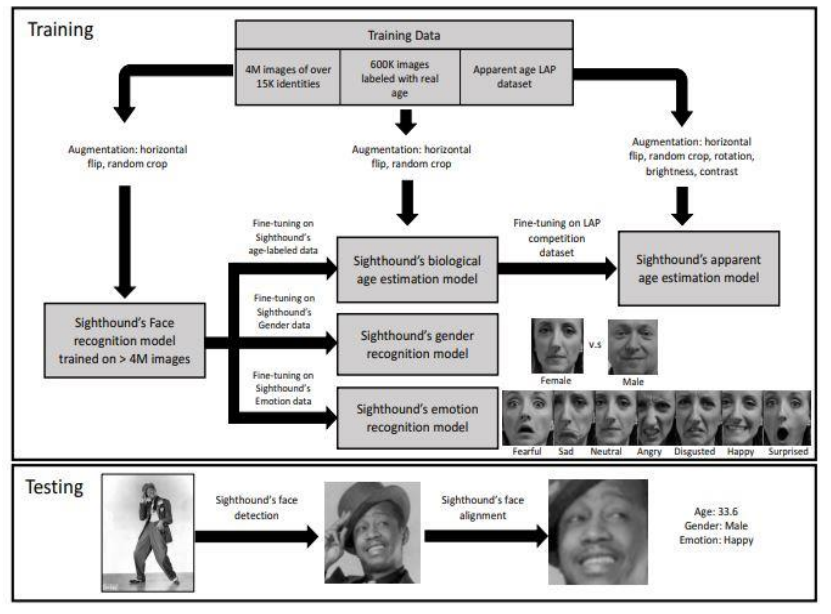

Figure 2: Flow Proposed methodology.

\section{Feature Classification}

The classification stage recognizes facial images and group them according to certain classes and helps them Fig-2: Flow chart of proposed methodology skilled recognition. Classification is a complicated process because it can be playing role in many areas. Its classification stage also can call feature selection stage, which is deals with exchanges the retain essential information and connect them in certain parameters.

\section{E. Extraction And Classification Method}

1. SVM

It is an AI calculation which is utilized for grouping and relapse. Yet, as a rule, they are utilized for grouping issues. SVM was first presented in the 1960s, however it was idealized in 1990. Contrasted and other AI calculations, SVM has its novel execution. The model is fundamentally a portrayal of various classes in a hyper plane of a multidimensional space. SVM will create hyper planes in an iterative way, which can limit blunders. The objective of SVM is to separate the informational index in toa few classifications to track down the most extreme edge hyper plane (MMH).

\section{LBP}

The Local Binary Pattern was utilized for surface characterization, later it was applied in other application. LBP relegates picture pixel by the area (p) every pixel esteem inside the sweep (R), addressed by (gp) taking the focal esteem $(\mathrm{gc})$ as the limit and changing over its edge 
into a given decimal number mark by eq. The principle property of LBP is its computational effortlessness, which make it dissecting conceivable a picture continuously.

\section{Hoard}

Histogram of arranged slope is highlight descriptor. Highlight descriptor is portrayals of pictures or Image patches, which work on the way toward extricating helpful data from pictures and disposing of excess data. Which was proposed for object identification in different AI application likewise PC vision. Hoard descriptor mostly centered around the design of a picture or object by tallying the confined segment. It is an element extraction thick strategy for pictures. Thick implies that it removes highlights from all situations in the picture (or then again locales of interest in the image), instead of just key focuses like SIFT in the nearby area.

\section{PCA}

PCA calculation is an eigenvector strategy to show direct variety of high dimensional information. Head part examination (PCA) is the most famous appearance-based factual strategy utilized predominantly for dimensionality decrease in pressure and acknowledgment. The PCA procedure is presented by Kirby in 1988. This method is utilized for dispose of the element of a face space information. The decrease of measurement assists with eliminating the insignificant data from input pictures to perceive the face. The principal face pictures can be presented as an element vector or weighted amount of the Eigen's appearances and put away in one - dimensional cluster. The PCA requires full front facing face picture to be introduced each an ideal opportunity to give the great exhibition. The benefit of PCA is it can lessen the necessary information to distinguish people to $1 / 1000$ of the showed information.

\section{Viola-Jones Algorithm}

The viola-Jones calculation primarily utilized for object identification work that can supply a serious pace of item recognition continuously application. Likewise, it tends to be prepared to distinguish the article classes likewise face discovery. Three significant commitments/period of this calculation are including extraction, characterization utilizing boosting, multi-scale discovery calculation. The principle benefit of viola-jones calculation, it is very quick include calculation and viable component choice.

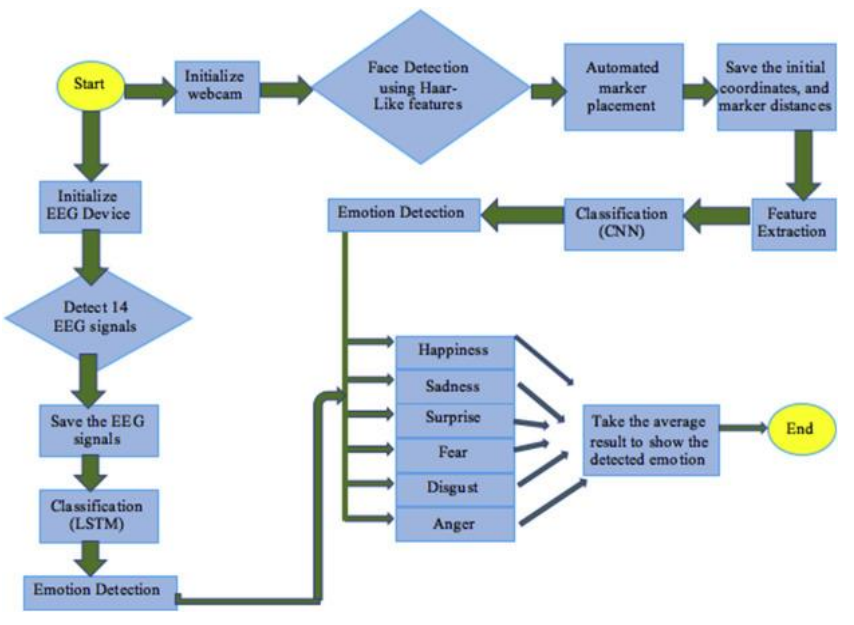

Figure 3: System Block Diagram.

\section{DATA COLLECTION}

Information collection assumes a substantial element in making ready any profound neural employer (DNN). In this paper, we suggest to mark facts for 3 separate errands: age, gender and feeling acknowledgment. Gathering marked facts for positive errands, like real age assessment, is substantially extra checking out contrasted with mainstream arrangement or recognition issues. This specialty is due to the manner that human mistake in assessing real age is large (now and then extra noteworthy than the PC imaginative and prescient assessments) and one cannot rely on human annotators to mark faces with their bearing on real age. In any case, at Sighthound we have accumulated a large dataset of countenances with their bearing on age, gender and feeling marks. As a way as every person is concerned, our datasets are the biggest or amongst the largest in both the scholastic or commercial enterprise world [6]. Underneath we provide some insights at the facts applied for making ready our fashions.

\section{A. Face reputation}

The base version for our facial acknowledgment is ready on greater than $4,000,000$ pix of greater than 40,000 people. The large range in pix of each character make our 
profound version full of life to normal problems in face acknowledgment. Our face acknowledgment version is offered to designers via the Sighthound Cloud API.

\section{B. Age estimation}

As of overdue there had been some endeavors in accumulating data with evaluating age names Among those, the dataset proposed with an id of using Rothe. In this most important dataset that includes 523, 051 pix what is greater, is offered for studies purposes. In any case, the dataset isn't always cautiously defined and includes several mix-ups. Also, the dissemination of the data throughout diverse a long time is pretty lopsided. This brought on the creators utilizing simply $50 \%$ of the data for getting ready withinside the first paper. To higher deal with this tough we collected a large dataset of 600,000 pix with evaluating age names. As against beyond works, our dataset has a greater adjusted circulate throughout diverse a long time. For example, we've over 120,000 people in our dataset with named a long time greater than 70 or greater younger than twenty years old. We applied a collection of human annotators to extra smooth our dataset via a semi-controlled system.

\section{Gender and emotion reputation}

Our 4,000,000 countenances marked for the errand of face acknowledgment are likewise named with their concerning gender orientation. To higher enhance our version, we delivered a large wide variety of pix of diverse identities simply as age gatherings. Moreover, we additionally clarified a part of our data with feeling marks for the errand of feeling acknowledgment.

\section{Data pre-processing}

We pre-method every photo previous to looking after them to our DNNs. These pre-handling steps include face discovery, facial milestone reputation and arrangement. We applied Sighthound's face reputation which is offered via our cloud API. In the occasion that a couple of face is diagnosed in a photo, we select out the maximum centered one. (This is specifically the scenario withinside the
ChaLearn v2 dataset which includes one-of-a-kind appearances). In the Cha learn dataset we had the choice to discover all faces utilizing a mix of methods, but all utilizing the Sighthound Cloud APIs. Given the face leaping boxes, we recognize [7].

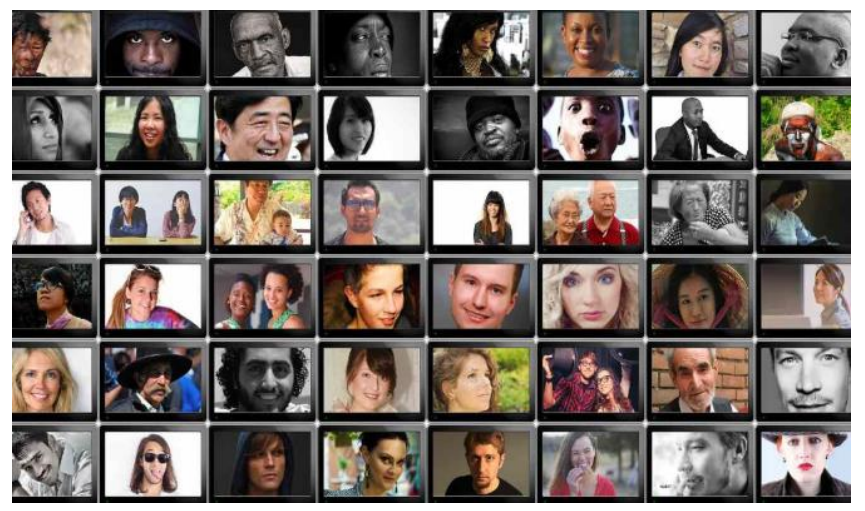

Fig. 2: Qualitative effects of our approach on ChaLearn LAP challenge

The pinnacle part of the determine suggests a few instance snap shots wherein our excellent blunder isn't precisely a large part of a year furthermore, the bottom 1/2 of suggests snap shots wherein our blunder changed into over 5 years. As appeared, the presentation of our method drops for the maximum component for dim scale/oldfashion snap shots. Rather than a few beyond works, which do not make use of any face arrangement, we determined this to be sizable in our ultimate precision numbers. Deep training: As established in Figure 1, we begin through making ready a profound neural community for the venture of face acknowledgment using 4,000,000 snap shots of over 40, 000 characters. Our face acknowledgment version isn't always simply computationally cheap (with consist of extraction season of $70 \mathrm{~ms}$ using handiest the CPU), but likewise accomplishes awesome effects at the LFW dataset. This version serves as the muse of our facial assets acknowledgment motor. We deliberate an exceptionally streamlined profound employer engineering for exactness and velocity for every task. In a few new works, experts try to plot an employer which performs out all undertakings simultaneously, and that they have proven minor upgrades. Nonetheless, having separate businesses for every venture accredited us to plot faster and extra versatile fashions for every errand. Also strolling all fashions joined takes much less time contrasted with the throughout the board version 
of what is extra, we accomplish higher effects. We ought to add that notwithstanding the truth that the employer engineering isn't always something comparable for every errand, all businesses are organized first for the errand of facial acknowledgment using the 4,000,000-image set.

The modules of the work are represented in following figures.

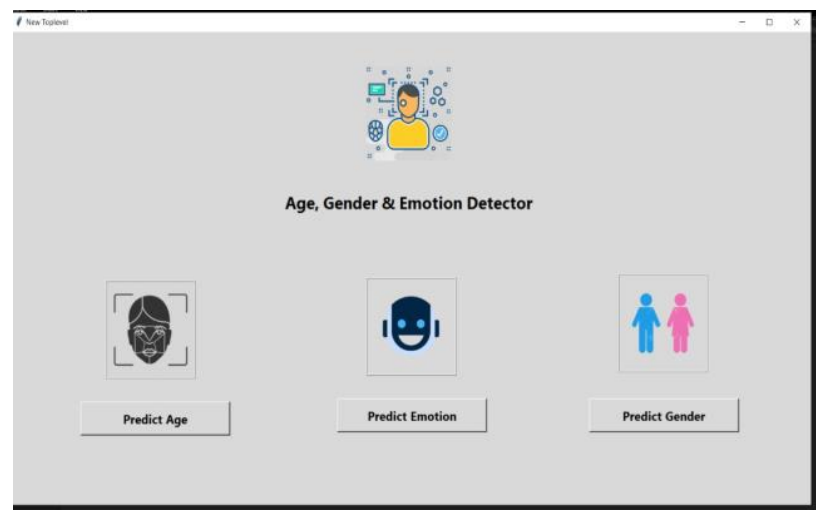

Figure 4: Age predictor

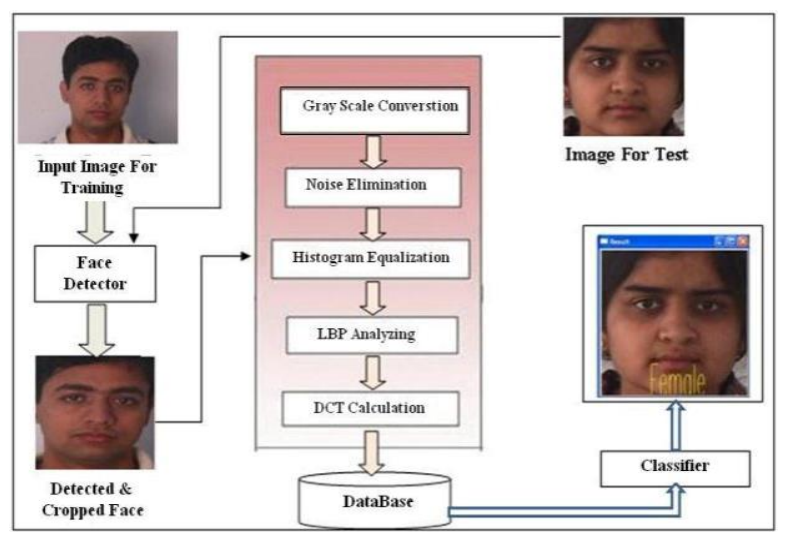

Figure 5: Gender identification

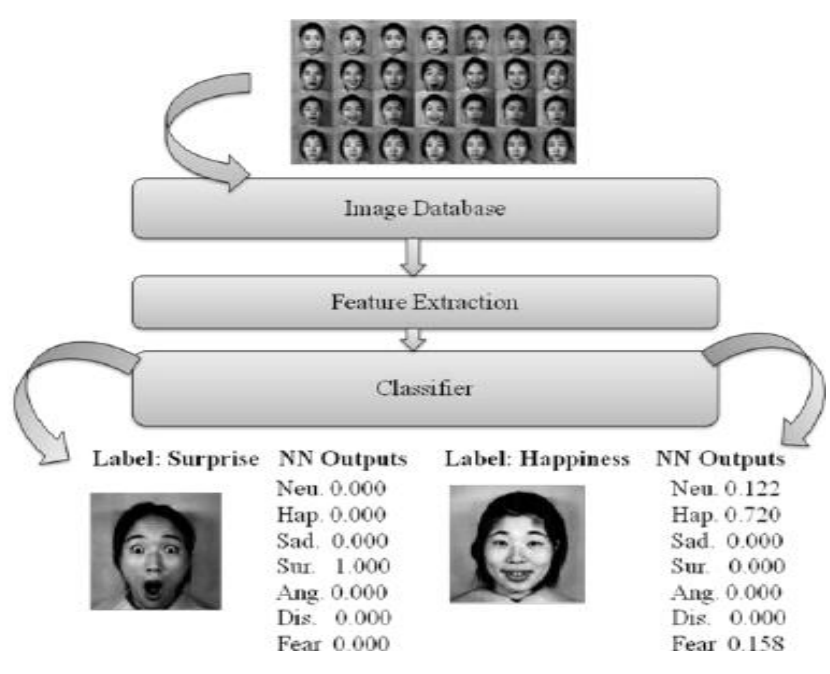

Fig. 6 Emotion Detection.
Media Sources - In this module it provides facilities to choose different sources of input such as webcam or image.

\section{CONCLUSIONS}

In view of this investigation a total review of the condition for age, gender and feeling characterization has been assessed and talked about by means of face pictures. Face pictures have gotten significant in late many years basically due to their promising true application in a few arising fields.

A general investigation of the commitment made on gender orientation, feeling grouping, and age assessment used to tackle the continuous application issues and its application regions are measurable, security, face identification amalgamation, lie discovery music for mindset, mechanized mentoring frameworks. In this paper a large portion of the exploration work is done is in Convolutional Neural Organization and calculation like, AdaBoost, Hoard, HAAR, LBP, PCA, FPLBP, and LDA which are utilized for age, gender and feeling acknowledgment.

\section{REFERENCES}

[1] Mohammed Kamel Benkaddour, Sara Lahlali, Maroua Trabelsi, "Human Age and Gender Classification using Convolutional Neural Network", 2nd International Workshop on Human-Centric Smart Environments for Health and Well-being (IHSH), IEEE Xplore, 9-10 Feb. 2021. DOI: 10.1109/IHSH51661.2021.9378708

[2] Mossaad Ben Ayed; Sabeur Elkosantini; Shaya Abdullah Alshaya; Mohamed Abid, "Suspicious Behavior Recognition Based on Face Features", IEEE Access, Volume: 7, 14 October 2019, pp. 149952 - 149958.

DOI: 10.1109/ACCESS.2019.2947338

[3] Maciej Szankin; Alicja Kwasniewska; Jacek Ruminski, "Influence of Thermal Imagery Resolution on Accuracy of Deep Learning based Face Recognition", 12th International Conference on Human System Interaction (HSI), IEEE Xplore, 25-27 June 2019.

DOI: 10.1109/HSI47298.2019.8942636

[4] Iftikhar Alam; Shah Khusro, "Tailoring Recommendations to Groups of Viewers on Smart TV: A Real-Time Profile 
Generation Approach", IEEE Access, Volume: 8, 11 March 2020, pp. $50814-50827$.

DOI: 10.1109/ACCESS.2020.2980206.

[5] Razieh Rezaee; Abbas Ghaemi Bafghi; Masoud KhosraviFarmad, "A threat risk estimation model for computer network security", 6th International Conference on Computer and Knowledge Engineering (ICCKE), IEEE Xplore, 20-20 Oct. 2016.

DOI: 10.1109/ICCKE.2016.7802144.

6. Liying Lang; Yue Hong, "The Application of Face Recognition in Network Security", International Conference on Computational Intelligence and Security, IEEE Xplore, 13-17 Dec. 2008. DOI: 10.1109/CIS.2008.25

7. Dwi Ana Ratna Wati; Dika Abadianto, "Design of face detection and recognition system for smart home security application", 2nd International conferences on Information Technology, Information Systems and Electrical Engineering (ICITISEE), IEEE Xplore, 1-2 Nov. 2017.

DOI: 10.1109/ICITISEE.2017.8285524. 